\title{
Accelerated versus delayed initiation of renal-replacement strategies following cardiac surgery
}

\author{
HelenMari Merritt-Genore, DO, ${ }^{a}$ Kathleen M. Sarber, MD, ${ }^{b}$ and Shaun Thompson, MD $^{\mathrm{c}}$
}

Feature Editor Note-Cardiac surgery-associated acute kidney injury (AKI) is common and has significant prognostic implications, including the substantial long-term effects of persistent renal failure and a clear impact in mortality. Moreover, fluid overload is a frequent consequence of perioperative resuscitation in the early stages of recovery after cardiac surgery, specifically after complex procedures with massive fluid shifts. An important component of the approach to manage severe AKI is the use of renal-replacement therapy (RRT). Timing of initiation of RRT for AKI with or without volume overload is a controversial dilemma that we encounter frequently in perioperative cardiothoracic care. Broadly speaking, there is no difference in mortality when evaluating accelerated or early $R R T$ initiation versus delayed or only starting RRT when absolutely indicated in critically ill patients, and data in cardiac surgery are conflicting at best. There may be benefit from early RRT in subsets of cardiac surgical patients, but also there appears to be a significant number of patients who recover renal function in whom initiation of RRT could be detrimental or counterproductive.

In this invited expert opinion paper, Dr Merritt-Genore and colleagues review this controversial and timely topic. The authors start by acknowledging the different definitions of AKI and the fact that currently there are no clear tools to determine whether AKI is likely to be transient or persistent at time of diagnosis, data that would help determine when would be best to have a careful watchful approach versus early RRT. The authors go on to review the data for the timing of RRT in critically ill patients in general, followed by segments specific to cardiac surgery and special patient populations, such as those with left ventricular assist devices, heart transplant recipients, and those receiving extracorporeal membrane oxygenation complicated with AKI.

The debate is not settled. It is clear that having more tools to determine the probability for persistent AKI would help solve the controversy to ideally provide RRT at the right time to the right patient.

\section{Juan N. Pulido, MD}

From ${ }^{\mathrm{a}}$ Heart Consultants, Methodist Physicians Clinic, Omaha, Neb; ${ }^{\mathrm{b}}$ Department of Surgery, F. Edward Hebert School of Medicine, Uniformed Services University of the Health Sciences, Bethesda, Md; and ${ }^{\mathrm{c}}$ Department of Anesthesiology, University of Nebraska Medical Center, Omaha, Neb.

Received for publication March 12, 2021; accepted for publication March 12, 2021. Address for reprints: HelenMari Merritt-Genore, DO, 1120 N 103rd Pl, Omaha NE 68114 (E-mail: helenmari.merrit@gmail.com).

JTCVS Open 2021;6:193-7

2666-2736

Copyright $\odot 2021$ The Author(s). Published by Elsevier Inc. on behalf of The American Association for Thoracic Surgery. This is an open access article under the CC BY-NC-ND license (http://creativecommons.org/licenses/by-nc-nd/4.0/).

https://doi.org/10.1016/j.xjon.2021.03.023

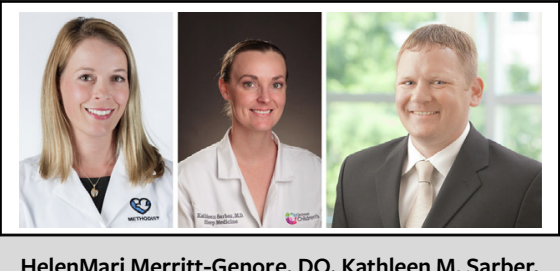

HelenMari Merritt-Genore, DO, Kathleen M. Sarber,

MD, and Shaun Thompson, MD

CENTRAL MESSAGE

The initiation of early renalreplacement therapy for acute kidney injury following cardiac surgery remains controversial, with data supporting both accelerated and delayed strategies.

See Commentaries on pages 198 and 200.

Acute kidney injury (AKI) is known to increase length of hospital stay, morbidity, and mortality following cardiac surgery. Multiple definitions exist for the designation and classification of AKI. For instance, the Society of Thoracic Surgeons establishes acute renal failure as a 2 -fold rise in serum creatinine $(\mathrm{SCr}$ ), or $\mathrm{SCr}>4.0 \mathrm{mg} / \mathrm{dL}$ (with a minimum rise of $0.5 \mathrm{mg} / \mathrm{dL}$ ), or new hemodialysis. ${ }^{1}$ The definition of AKI from Kidney Disease: Improving Global Outcomes, in contrast, includes an increase in $\mathrm{SCr}$ by $>0.3 \mathrm{mg} / \mathrm{dL}$ within 48 hours or an increase within 7 days' time of baseline $\mathrm{SCr}$ by $>1.5$ times or urine output $<0.5 \mathrm{~mL} / \mathrm{kg} / \mathrm{h}$ for 6 hours, and further characterizes the stage of AKI to more granular levels. While the criteria to define AKI vary, ${ }^{2}$ the incidence of kidney injury remains high in cardiac surgery, with between $4 \%$ and $35 \%$ of patients experiencing some form of $\mathrm{AKI}^{3-5}$ and between $2 \%$ and $20 \%$ of patients requiring renal-replacement therapy (RRT) in the postoperative period. In this population, RRT is independently associated with an up to 8-fold increase in mortality. ${ }^{4}$

The causes of AKI after cardiac surgery are many. In addition to known preoperative risk factors (pre-existing renal dysfunction, recent contrast, diabetes, advanced age), perioperative myocardial injury and fluctuations in cardiac output may lead to increased sympathetic activity, increased production of vasopressin, and activation of the 
renin-angiotensin-aldosterone system. Added to this are the effects of the cardiopulmonary bypass circuit on arterial resistance and subsequent volume retention postoperatively. All of this may create a vicious cycle of repeated kidney injury with renal sodium and water retention and extracellular fluid expansion. Perioperative fluid overload is associated with worse outcomes and is a primary risk factor for multiorgan failure, including acute renal failure, ${ }^{6}$ yet the timing and intensity of RRT in the postoperative cardiac patient remains controversial, with many factors playing into the decision for initiation, such as acidosis, urine output, and fluid balance, and the overall clinical picture (Figure 1). In addition, there are few data to guide the clinician's determination as to whether the AKI is transient and retains capacity for early recovery or whether the injury will progress to azotemia and volume overload, perhaps despite a lower SCr. In this Expert Review, we will examine and summarize contemporary studies focusing on accelerated versus delayed strategies for RRT following cardiac surgery. As the bulk of high-quality studies come from critically ill populations (and not specifically cardiac surgery patients), we will examine the data separately and discuss what can be generalized to individual populations.

\section{CRITICALLY ILL PATIENTS}

Contemporary studies examining the impact of the timing of RRT have tended to focus on critically ill patients, which may or may not include cardiac surgical patients specifically. The studies have generally concentrated on survival benefit and recovery of renal function as primary outcomes. A large 2020 meta-analysis including 10 randomized controlled trials (RCTs) and 2143 critically ill patients with severe AKI found that in the absence of urgent indicators for RRT, there was no survival benefit to early RRT initiation. Somewhat surprisingly, this study also showed that up to $42 \%$ of patients in the late RRT group had recovery of renal function and never went on to require dialysis. $^{7}$ A second recent large meta-analysis of 18 RCTs confirmed similar findings and reinforced the idea that a delayed strategy may obviate the need for RRT in critically ill populations. ${ }^{8}$

The recently published STandard versus Accelerated initiation of Renal Replacement Therapy in Acute Kidney Injury (STARRT-AKI) multinational RCT randomized nearly 3000 critically ill patients (including 230 cardiac surgery patients) into early RRT or standard management of AKI, in which RRT was discouraged unless standard criteria for initiation were met. ${ }^{9}$ The primary end point of death at 90 days was similar between groups $(43.9 \%$ vs $43.7 \%, P=.92$ ). Prolonged RRT was observed at greater rates in 90-day survivors in the accelerated strategy group $(10.4 \%$ vs $6 \%$; relative risk, $1.74 ; 95 \%$ confidence interval [CI], 1.24-2.43). While the STARRT-AKI trial did include 230 patients who underwent cardiac surgery (accelerated $[\mathrm{n}=112]$; standard $[\mathrm{n}=118]$ ), there has not yet been a subgroup analysis of the cardiac surgery group. Significant heterogeneity in the population may limit the applicability of the results; thus, it is difficult to discern outcomes differences in cardiac surgery patients specifically based on the results of the STAART AKI trial.

Selected studies have also examined the effect of fluid balance in critically ill populations regarding the timing

Renal Replacement Therapy after Cardiac Surgery

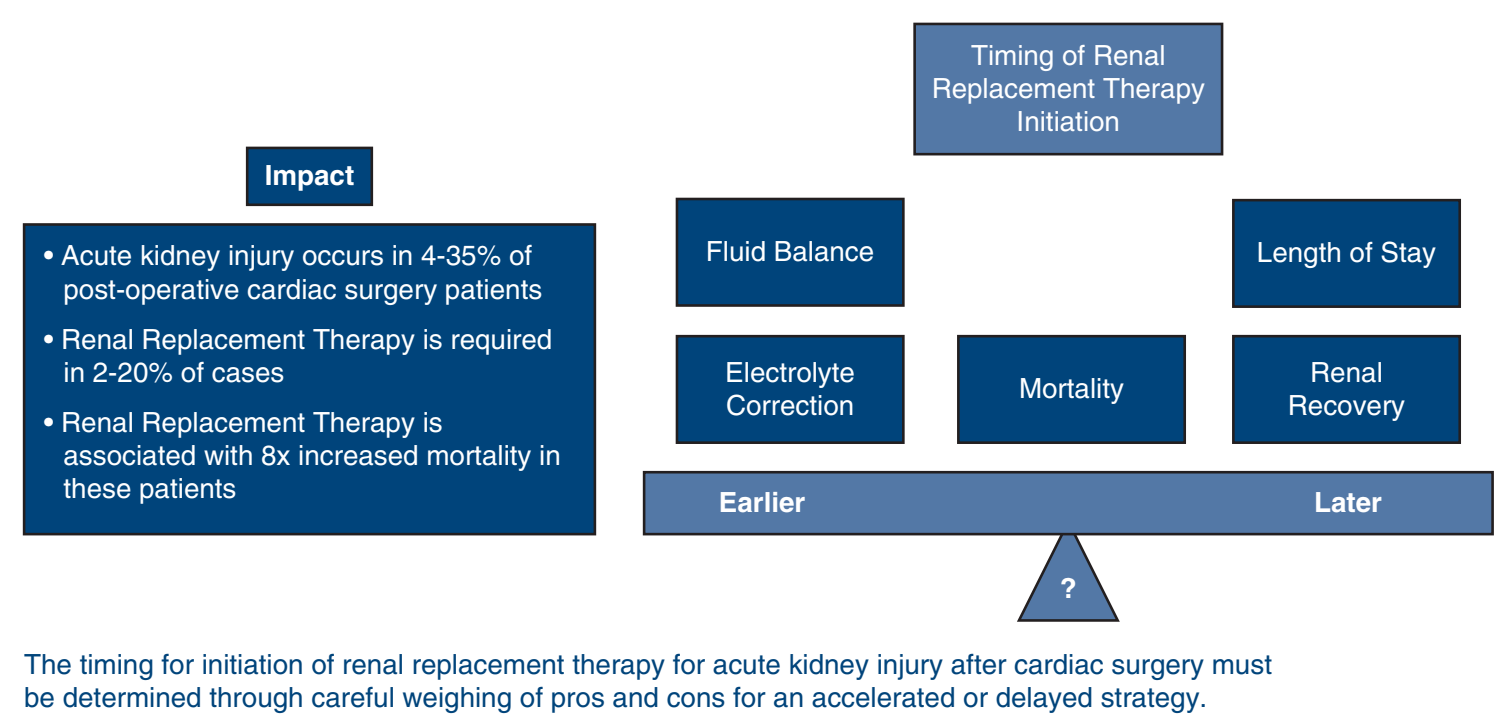

FIGURE 1. Visual representation of the impact and controversy surrounding the timing of initiation for renal-replacement therapy for acute kidney injury after cardiac surgery. On the left side of the image is the impact of acute kidney injury on postoperative heart surgery patients. On the right are the considerations a provider must undertake when determining the appropriating timing for renal-replacement therapy for individual patients. 
of RRT initiation. In a large retrospective study of 18,084 critically ill patients with AKI from various etiologies, ${ }^{10}$ positive fluid balance was associated with increased shortand long-term mortality (adjusted hazard ratio, 1.3-1.92) compared with even fluid balance. Negative fluid balance did show increased mortality risk using Gray's statistical model but not using logistic regression analysis. Interestingly, recovery of renal function was similar between all groups. A contrasting prospective study of 618 critically ill patients demonstrated fluid overload $(>10 \%$ body weight) at the time of RRT initiation was associated with odds ratio of death of 2.07 , and that patients with fluid overload at the time of peak creatinine were less likely to recovery kidney function long-term. ${ }^{11}$

\section{CARDIAC SURGERY PATIENTS}

There are fewer studies that specifically focus on the timing of RRT in cardiac surgery patients, and conclusions are somewhat conflicting. For instance, a 2016 retrospective study by Yang and colleagues ${ }^{12}$ included intraoperative and postoperative factors in the selection of patients for preemptive and standard RRT and found that a pre-emptive strategy had reduced rates of mortality. These findings were related to reducing fluid overload with early RRT compared with the standard treatment group. A 2014 meta-analysis of 841 cardiac surgery patients also reported a lower mortality in patients who received earlier RRT as compared with standard therapy (odds ratio [OR], 0.29; $95 \%$ CI, 0.16-0.52, $P<.0001$ ), and suggested a trend toward shorter length of stay in the intensive care unit (ICU). This meta-analysis included studies dating back as far as 1950, however, and a large degree of heterogeneity was observed, making generalization difficult. ${ }^{13}$

Another large contemporary meta-analysis ${ }^{14}$ included 1479 cardiac surgery patients in 15 different studies and concluded that AKI treated with early RRT had decreased 28-day mortality (OR, 0.36 ; 95\% CI, 0.23-0.57) and shortened ICU and hospital length of stay. A subgroup analysis was performed comparing the outcomes of cohort studies with the 5 RCTs. While the cohort studies supported the benefit of early RRT, the RCT analysis did not show a statistically significant decrease in mortality (OR, 0.41; 95\% CI, 0.14-1.24). There was also an earlier meta-analysis ${ }^{15}$ in 2008 that performed a subgroup analysis of RCTs and came to a similar conclusion that there was no survival benefit with earlier RRT initiation (RR, $0.64 ; 95 \% \mathrm{CI}$, $0.64-1.05 ; P=.08)$.

How do we reconcile this difference and apply these data to our own patients? Dropout bias frequently seen in cohort studies can negatively impact studies looking at an intervention in regards to timing, because patients likely have a better prognosis if their disease process improves before initiation of the intervention. This is illustrated in the largest
RCT on cardiac surgical patients to date, where more than one third of patients in the delayed RRT arm survived without ever needing RRT. ${ }^{16}$ In 2015, Crescenzi and colleagues ${ }^{17}$ attempted to avoid the dropout bias by prospectively enrolling 1658 cardiac surgery patient at the time of ICU admission, before any signs of postoperative renal insult. A total of 56 patients (3.6\%) required RRT after cardiac surgery within this study. Patients in the "late" group (oliguria for $>12$ hours) required significantly less RRT than in the early group (oliguria for $>6$ hours), highlighting again the possibility that patients may recover renal function before requiring RRT. There was no significant difference in mortality or length of stay in the ICU or hospital between groups. Importantly, the authors looked at the "late" group and determined that 21 patients who did not start RRT would have actually received RRT had they been in the "early" group, as the duration of their oliguria would have met criteria. Although overall mortality was similar between groups, a survival benefit was observed for a subset of patients with pre-existing renal dysfunction in the "late" RRT group. This may suggest a benefit in "watchful waiting" for patients undergoing cardiac surgery with preoperative renal dysfunction, but more work is needed in this area.

Each of these trials use different criteria, such as urine output, SCr, and blood urea nitrogen for initiation of RRT. Even the definition of "early" versus "late" initiation of RRT varies significantly between studies, with some trials using time from decreased urine output versus time after acute renal failure was diagnosed. Use of these parameters does not allow many other complex factors to be considered. Importantly and specifically in the cardiac postoperative period, these factors often drive AKI but are not traditional indicators for RRT. In patients undergoing cardiac surgery, volume shifts are common in postoperative recovery and are traditionally managed by use of diuretics expectantly. When renal function is compromised, excessive volume retention may lead to deleterious effects in a depressed left or right ventricle and thus some have advocated for earlier initiation of RRT to diminish these effects.

\section{SPECIAL CIRCUMSTANCES}

The development of AKI in postoperative heart transplant patients is also a serious concern, with up to $12 \%$ of patients requiring peritransplant RRT, and an association with greater hospital and 1-year mortality in these patients. ${ }^{18}$ Shen and colleagues ${ }^{19}$ presented a retrospective study of 76 patients over 10 years' time to determine optimal timing for initiation of RRT in cardiac transplant patients with AKI postoperatively. Patients with earlier RRT had significantly lower mortality $(39.1 \%$ vs $63.3 \%$, $P=.039$ ), shorter ICU and hospital lengths of stay, and lower overall cost. Contrary to some of the previous studies, 
recovery of renal function was more common in the accelerated RRT group than for the delayed cohort in this study. Likewise, a 2020 retrospective study by Liu and colleagues $^{20}$ used Kidney Disease: Improving Global Outcomes staging for AKI to determine the need for RRT in 184 patients with a left ventricular assist device. In this cohort, an "early" strategy was associated with a trend toward shorter ICU and hospital length of stay, as well as lower need for permanent RRT, with similar mortality between groups. Further work is needed in the area of transplantation and left ventricular assist devices, including high-quality prospective studies to examine the appropriate timing of initiating RRT to reduce preload in patients with an acute or chronically failing right ventricle.

There has also been the suggestion that RRT may help improve the prognosis of critically ill patients by filtering inflammatory markers in addition to managing volume overload. Several RCTs have examined RRT and immunomodulation in critically ill patients with sepsis. Targeted cytokine size is important, with many filtration membranes still allowing passage of macromolecules such as tumor necrosis factor-alpha, interleukin-6, and interleukin-1. Heterogeneity in patient populations and lack of uniformity of study designs have limited the applicability of singular studies, and the results remain somewhat contradictory. ${ }^{21,22}$ One RCT examining cardiac surgery patients with severe shock and renal dysfunction targeted RRT to remove proinflammatory mediators and reduce vasopressor requirements but found no significant differences in the RRT versus standard groups for mortality or renal recovery. ${ }^{16}$ These theories of cytokine reduction have been recently debated in the context of the coronavirus disease 2019 pandemic, and data are still emerging at this time.

Lastly, it has been well-established that renal dysfunction occurs commonly in patients supported on extracorporeal membrane oxygenation (ECMO). A large recent metaanalysis that included 5896 adult patients on ECMO demonstrated an $81 \%$ increased risk of death (RR, $1.81 ; 95 \% \mathrm{CI}$, 1.56-2.08, $P<.001)$ for patients who required new RRT while on support. ${ }^{23}$ Fluid balance has been identified as a predictor of mortality in patients receiving ECMO/RRT. ${ }^{24}$ Although there has been a trend toward improving survival in patients receiving ECMO/RRT over the past 20 years, further work is certainly needed in this realm to define indications for initiation of RRT and methods to decrease renal dysfunction in this complex population.

\section{CONCLUSIONS}

It is clear the long-term effect of AKI after cardiac surgery is substantial. While the use of RRT can substitute key portions of renal function and help maintain euvolemia in the immediate postoperative phase, mortality is greater in patients who suffer AKI after cardiac surgery regardless of whether RRT is used. However, patients who do not recover to their baseline renal function may have twice the longterm mortality risk than those who do recover. ${ }^{25}$ Increasing evidence from RCTs in critical care populations suggest a delayed strategy may allow renal recovery and a high rate of avoidance of long-term RRT dependence, ${ }^{7,8,11,16}$ without an increased risk of mortality.

Further high-quality studies within this specific cohort are needed before this debate can be settled. Future studies should look not only into the timing and modality of RRT used but also patient factors (ventricular and pulmonary function in addition to standard preoperative risk factors) and perioperative events (longer pump time, coagulopathy requiring blood transfusions, fluid balance, hypotension requiring inotropic support, expected postoperative course, and mechanical support), which may lead to the development of AKI and need for RRT. Presently, the timing of RRT after cardiac surgery is still a decision that continues to generate debate due to lack of consensus and requires an individualized and multidisciplinary approach.

\section{Conflict of Interest Statement}

The authors reported no conflicts of interest.

The Journal policy requires editors and reviewers to disclose conflicts of interest and to decline handling or reviewing manuscripts for which they may have a conflict of interest. The editors and reviewers of this article have no conflicts of interest.

\section{References}

1. O'Brien SM, Shahian DM, Filardo G, Ferraris VA, Haan CK, Rich JB, et al. The Society of Thoracic Surgeons 2008 cardiac surgery risk models: part 2-isolated valve surgery. Ann Thorac Surg. 2009;88(1 suppl):S23-42.

2. Dudar I, Loboda O, Savchuk V. KDIGO 2020 Clinical Practice Guideline for Diabetes Management in Chronic Kidney Disease: summary of recommendation statements and practice points translated into Ukrainian. Ukrainian J Nephrol Dial. 2020;68:59-66.

3. Bastin AJ, Ostermann M, Slack AJ, Diller G-P, Finney SJ, Evans TW. Acute kidney injury after cardiac surgery according to Risk/Injury/Failure/Loss/End-stage, Acute Kidney Injury Network, and Kidney Disease: Improving Global Outcomes classifications. J Crit Care. 2013;28:389-96.

4. Ramos KA, Dias CB. Acute kidney injury after cardiac surgery in patients without chronic kidney disease. Braz J Cardiovasc Surg. 2018;33:454-61.

5. Hu J, Chen R, Liu S, Yu X, Zou J, Ding X. Global incidence and outcomes of adult patients with acute kidney injury after cardiac surgery: a systematic review and meta-analysis. J Cardiothorac Vasc Anesth. 2016;30:82-9.

6. Stein A, de Souza LV, Belettini CR, Menegazzo WR, Viégas JR, Costa Pereira EM, et al. Fluid overload and changes in serum creatinine after cardiac surgery: predictors of mortality and longer intensive care stay. A prospective cohort study. Crit Care. 2012;16:R99.

7. Gaudry S, Hajage D, Benichou N, Chaïbi K, Barbar S, Zarbock A, et al. Delayed versus early initiation of renal replacement therapy for severe acute kidney injury: a systematic review and individual patient data meta-analysis of randomised clinical trials. Lancet. 2020;395:1506-15.

8. Zhang L, Chen D, Tang X, Li P, Zhang Y, Tao Y. Timing of initiation of renal replacement therapy in acute kidney injury: an updated meta-analysis of randomized controlled trials. Ren Fail. 2020;42:77-88.

9. STARRT-AKI Investigators, Canadian Critical Care Trials Group, Australian and New Zealand Intensive Care Society Clinical Trials Group, United Kingdom Critical Care Research Group, Canadian Nephrology Trials Network, Irish 
Critical Care Trials Group, et al. Timing of initiation of renal-replacement therapy in acute kidney injury. $N$ Engl J Med. 2020;383:240-51.

10. Balakumar V, Murugan R, Sileanu FE, Palevsky P, Clermont G, Kellum JA. Both positive and negative fluid balance may be associated with reduced long-term survival in the critically ill. Crit Care Med. 2017;45:e749-57.

11. Bouchard J, Soroko SB, Chertow GM, Himmelfarb J, Ikizler TA, Paganini EP, et al. Program to Improve Care in Acute Renal Disease (PICARD) Study Group Fluid accumulation, survival and recovery of kidney function in critically ill patients with acute kidney injury. Kidney Int. 2009;76:422-7.

12. Yang X-M, Tu G-W, Gao J, Wang C-S, Zhu D-M, Shen B, et al. A comparison of preemptive versus standard renal replacement therapy for acute kidney injury after cardiac surgery. J Surg Res. 2016;204:205-12.

13. Liu Y, Davari-Farid S, Arora P, Porhomayon J, Nader ND. Early versus late initiation of renal replacement therapy in critically ill patients with acute kidney injury after cardiac surgery: a systematic review and meta-analysis. J Cardiothorac Vasc Anesth. 2014;28:557-63.

14. Zou H, Hong Q, Xu G. Early versus late initiation of renal replacement therapy impacts mortality in patients with acute kidney injury post cardiac surgery: a meta-analysis. Crit Care. 2017;21:150.

15. Seabra VF, Balk EM, Liangos O, Sosa MA, Cendoroglo M, Jaber BL. Timing of renal replacement therapy initiation in acute renal failure: a meta-analysis. Am J Kidney Dis. 2008;52:272-84.

16. Combes A, Bréchot N, Amour J, Cozic N, Lebreton G, Guidon C, et al. Early high-volume hemofiltration versus standard care for post-cardiac surgery shock. The HEROICS study. Am J Respir Crit Care Med. 2015; 192:1179-90.

17. Crescenzi G, Torracca L, Pierri MD, Rosica C, Munch C, Capestro F. "Early" and "late" timing for renal replacement therapy in acute kidney injury after cardiac surgery: a prospective, interventional, controlled, single-centre trial. Interact Cardiovasc Thorac Surg. 2015;20:616-21.
18. Thongprayoon C, Lertjitbanjong P, Hansrivijit P, Crisafio A, Mao MA, Watthanasuntorn K, et al. Acute kidney injury in patients undergoing cardiac transplantation: a meta-analysis. Medicines (Basel). 2019;6:108.

19. Shen B, Xu J, Lv W, Jiang W, Wang Y, Nie Y, et al. Efficacy of early goal-directed renal replacement therapy for the treatment of acute kidney injury after heart transplantation: a single-center 10-year experience. J Cardiothorac Vasc Anesth. 2020;34:1534-41.

20. Liu H, Jeng E, Demos D, Vilaro J, Ahmed MM, Parker A, et al. Early versus standard renal replacement therapy after left ventricular assist device implantation. $J$ Card Surg. 2020;35:2529-38.

21. Vaara ST, Bellomo R. Extra-renal indications for continuous renal replacement therapy. Contrib Nephrol. 2018;194:90-8.

22. Chen G, Zhou Y, Ma J, Xia P, Qin Y, Li X. Is there a role for blood purification therapies targeting cytokine storm syndrome in critically severe COVID-19 patients? Ren Fail. 2020;42:483-8.

23. Mitra S, Ling RR, Tan CS, Shekar K, MacLaren G, Ramanathan K. Concurrent use of renal replacement therapy during extracorporeal membrane oxygenation support: a systematic review and meta-analysis. J Clin $\mathrm{Med}$ Res. 2021;10:241.

24. Dado DN, Ainsworth CR, Thomas SB, Huang B, Piper LC, Sams VG, et al. Outcomes among patients treated with renal replacement therapy during extracorporeal membrane oxygenation: a single-center retrospective study. Blood Purif. 2020;49:341-7.

25. Corredor C, Thomson R, Al-Subaie N. Long-term consequences of acute kidney injury after cardiac surgery: a systematic review and meta-analysis. J Cardiothorac Vasc Anesth. 2016;30:69-75.

Key Words: critical care, renal-replacement therapy, ultrafiltration, postcardiotomy 